\title{
Development of New Variety in Cluster Bean (Cyamopsis tetragonoloba)
}

\author{
V. Premalakshmi*, T. Arumugam, N. Deepa Devi and S. Ramesh Kumar \\ Department of Vegetable Crops, HC\&RI, Tamil Nadu Agricultural University, \\ Coimbatore- 641003, India \\ *Corresponding author
}

\begin{tabular}{|c|c|}
\hline & B S T R A C T \\
\hline Keywo & \multirow{3}{*}{$\begin{array}{l}\text { Nine local and } 63 \text { exotic collections of Cluster bean (Cyamopsis tetragonoloba) were } \\
\text { collected from different geographical locations of India and evaluated their performance } \\
\text { for identification and selection suitable genotype for Tamil Nadu rain fed cultivation } \\
\text { Significant differences among accessions were found for all the traits studied Results } \\
\text { indicated that the Acc IC } 28269 \text {,IC } 421808 \text {,IC } 402293 \text {, and Virudhunagar local recorded } \\
\text { maximum height of the plant and Thadayampatti local, IC11354, IC } 421350 \text { and IC } \\
432117 \text { recorded medium height maximum number of branches were recorded in IC10350, } \\
\text { IC11704 IC } 134956 \text { followed by IC432117, IC } 402293 \text { and IC } 11354 \text { The yield parameters } \\
\text { viz., number of fruits per plant, average individual fruit weight and yield per plant with } \\
\text { long fruits and maximum girth of the fruit was recorded in IC } 432117 \text {, Virudhunagar local } \\
\text { and Thadayampatti local The above genotypes were identified as promising genotypes } \\
\text { suitable for grown under rainfed condition of Tamil Nadu. }\end{array}$} \\
\hline Ar & \\
\hline $\begin{array}{l}\text { vailable Online: } \\
0 \text { April } 2017\end{array}$ & \\
\hline
\end{tabular}

\section{Introduction}

Cluster bean (Cyamopsis tetragonoloba) is an important leguminous crop, highly adopted to arid and semi-arid parts of the requiring low inputs and care. It is cultivated mainly in rainy season as rain fed crop in arid zones. India various parts of the world (Pathak et al., 2009) In India the crop is mainly grown in the dry habitats of Rajasthan, Haryana, Gujarat and Punjab. In Tamil Nadu the total area of cultivation of cluster bean is 600ha and major cultivating districts are Thoothukudi (180ha), Madurai (100ha), Namakkal (44ha), Coimbatore (19ha), Cuddalore (17ha) and Virudhunagar (11ha). Since it is relatively tolerant to salt and drought and gives high yield under adverse conditions and needs less fertilization it is suitable for the small farmers and marginal farmers and also suitable to cultivate under problematic soil condition. The crop is known for its exceptionally high adaptation towards poor and erratic rains multiuse in cropping system and in industrial uses in many ways besides other social and dietary uses These qualities have made it must be favored crop of marginal farmers in arid areas In Tamil Nadu there is no specific variety available and farmers are used to cultivate the local types. The one early introduction "Pusa Naubahar" from IARI, New Delhi has made a good spread in the area in Tamil Nadu. However the yield potential has substantially go down. Hence, identification of suitable genotypes with better yield as well as pod quality is the need of the hour. Hence, collection of genotypes from wide geographical area would serve as a 
gene pool not only for selection one among them for better adaptability but it would also serve as a basic breeding material for hybridization and selection of progenies with better recombination of desirable traits Therefore the study was undertaken with major objectives of to develop short duration, determinate, high pod yielding with vegetable traits like tenderness, fibreless, attractive green color and seasonal adoptability

\section{Materials and Methods}

An investigation was carried out in cluster bean (Cyamopsis tetragonoloba) to develop a variety suitable for southern districts of Tamil Nadu. Totally seventy two genotypes were collected from different parts of India, it includes NBPGR, New Delhi, CAZRI, Jodhpur, Rajasthan, CIAH (ICAR), Bechwel, Bikener, GAU, Gujarat, HAU, Hissar and from different parts of Tamil Nadu were collected and evaluated. The experiment was conducted at Dept of Horticulture, Agricultural College and Research Institute, Madurai from 2010- 2011 during Kharif and Rabi season in randomized block design with three replications The spacing adopted was $60 \times 30 \mathrm{~cm}$ following the agronomic practices recommended by TNAU Observations on Plant height $(\mathrm{cm})$, number of branches per plant, days to $50 \%$ flowering, individual fruit weight $(\mathrm{g})$, yield per plant $(\mathrm{kg})$ fruit length $(\mathrm{cm})$ and fruit girth $(\mathrm{cm})$ were recorded from 10 randomly selected plants from each plot The data was subjected to the statistical analysis

\section{Results and Discussion}

Evaluation of genotypes was accomplished based on their mean (per se) performance. The analysis of variance indicated that significant differences among the parents, which indicated that the genotypes are generally diverse The genotype Virudhunagar local recorded the highest plant height of 112 $\mathrm{cm}$ followed by IC 402293, IC 421802, IC 28269 and HG $258(95.2,94.3,93.8$ and 90.0 $\mathrm{cm})$ respectively Plant height is an important trait by which growth and vigor of the plants are measured The lowest plant height was recorded in IC $311438(39.2 \mathrm{~cm})$, IC 373464 $(43.4 \mathrm{~cm})$, followed by IC 311440, IC 10339 and IC320936 (43.8, 45.8 and $46.6 \mathrm{~cm})$ respectively, whereas plant height was maximum among the 72 genotype was. There existed variations in number branches per plant in genotypes studied An increase in the number of laterals per plant would facilitate production of more number of flower clusters leading to higher yield The study also showed that significant differences among the genotypes for number of branches per plant. Number of branches per plant among the genotype was 5-10 per plant The accessions viz., IC432117, IC402293, IC1135 and IC 381 recorded 8.0, 8.2, 8, 4, 8.8 and 10.20 branches per plant Singh et al., (1990) observed complementary gene action for branching and they suggested that in breeding program large population of segregating generations should be grown to recover enough regular cluster bearing plants. Gipson and Balakrishnan (1990) reported that estimated heritability was greater for pods per cluster followed by branches per plan Early flowering is one of the main attributes manifested in the hybrids The earliest genotype was IC 329062 (19 days) and IC 369838 (21 days). In cluster bean fruit length is one of the desirable character for vegetable purpose Among the 72 genotypes IC432117 has recorded pod length of $12.86 \mathrm{~cm}$ followed by Madurai local $2(13.30 \mathrm{~cm})$, Sattur local $(13.98 \mathrm{~cm})$, Pusa Naubahar, Thadayampatti local, and Virudhunagar local recorded the pod length of $11.76,12.75$ and $12.22 \mathrm{~cm}$. 
Table.1 Mean performance of cluster bean genotypes for growth and yield characters

\begin{tabular}{|c|c|c|c|c|c|c|c|c|c|}
\hline S.No. & Genotypes & $\begin{array}{c}\text { Plant } \\
\text { height } \\
\text { (cm) }\end{array}$ & $\begin{array}{c}\text { Number } \\
\text { of } \\
\text { branches }\end{array}$ & $\begin{array}{c}\text { Days to } \\
50 \% \\
\text { flowering }\end{array}$ & $\begin{array}{c}\text { Fruit } \\
\text { length } \\
(\mathbf{c m})\end{array}$ & $\begin{array}{l}\text { Fruit } \\
\text { girth } \\
(\mathrm{cm})\end{array}$ & $\begin{array}{c}\text { Number } \\
\text { of fruits } \\
\text { per } \\
\text { plant }\end{array}$ & $\begin{array}{c}\text { Individual } \\
\text { fruit } \\
\text { weight (g) }\end{array}$ & $\begin{array}{c}\text { Fruit } \\
\text { yield } \\
\text { per } \\
\text { plant } \\
(\mathrm{g}) \\
\end{array}$ \\
\hline 1. & IC 258101 & $64.00 *$ & $5.00 *$ & $31.40 *$ & 6.60 & 3.10 & 71.80 & 1.97 & 142.10 \\
\hline 2. & IC 285171 & $57.00 *$ & 2.70 & $32.20 *$ & 9.40 & 3.24 & 60.80 & $2.26^{*}$ & 145.41 \\
\hline 3. & IC 311407 & $47.60 *$ & 2.40 & $31.80 *$ & 7.20 & 3.10 & 82.60 & 1.26 & 136.68 \\
\hline 4. & IC 311438 & $39.20 *$ & 2.80 & $25.23 *$ & 6.06 & 3.10 & $115.20^{*}$ & 1.30 & 141.21 \\
\hline 5. & IC 311440 & $43.80 *$ & 3.20 & $28.90 *$ & 4.86 & 2.94 & 64.80 & $2.87 *$ & 135.73 \\
\hline 6. & IC 329036 & $46.60 *$ & 2.40 & $27.20 *$ & 3.70 & 3.20 & 44.00 & $2.30 *$ & 135.20 \\
\hline 7. & IC 329062 & 87.20 & $7.20 *$ & $19.40 *$ & 6.20 & 3.00 & $129.80^{*}$ & 0.49 & 103.13 \\
\hline 8. & IC 369838 & $64.60 *$ & $4.80 *$ & $21.00 *$ & 6.16 & 3.44 & $119.00^{*}$ & 1.36 & 105.19 \\
\hline 9. & IC 370496 & $52.60 *$ & 6.60 & 34.60 & 5.40 & 1.98 & $145.80^{*}$ & 0.92 & 103.89 \\
\hline 10. & IC 370509 & $47.20 *$ & 4.70 & $25.40 *$ & 4.50 & 3.30 & 55.60 & 1.15 & 148.51 \\
\hline 11. & IC 373438 & $51.00 *$ & 3.80 & $25.00 *$ & 5.92 & 3.18 & 46.20 & 1.59 & 152.98 \\
\hline 12. & IC 373464 & $43.40 *$ & 1.80 & $25.40 *$ & 5.10 & 2.42 & 60.20 & $3.27 *$ & 147.51 \\
\hline 13. & IC 402293 & 95.20 & $5.40 *$ & $21.40 *$ & 6.08 & 2.94 & $113.20^{*}$ & 1.40 & 180.55 \\
\hline 14. & IC 402296 & $69.40 *$ & $6.60 *$ & $23.40 *$ & 6.30 & 3.08 & $113.00^{*}$ & $2.76^{*}$ & 184.16 \\
\hline 15. & IC 402298 & 76.40 & $6.00 *$ & $27.40 *$ & 6.54 & 3.16 & $139.40^{*}$ & 1.42 & 193.26 \\
\hline 16. & IC 415106 & $55.40^{*}$ & $5.20 *$ & $27.00^{*}$ & 4.53 & 2.18 & 71.10 & 0.93 & 96.25 \\
\hline 17. & IC 415108 & $58.00 *$ & 3.70 & $23.50 *$ & 4.72 & 3.08 & 109.20 & 1.25 & 101.77 \\
\hline 18. & IC 415125 & $62.00 *$ & 3.90 & $24.50 *$ & 5.44 & 3.06 & 84.40 & 1.20 & 105.61 \\
\hline 19. & IC 415140 & $48.00 *$ & 3.50 & $25.40 *$ & 6.12 & 3.08 & 147.40 & 1.20 & 62.17 \\
\hline 20. & IC 421805 & 82.60 & 2.90 & $29.40 *$ & 5.72 & 2.98 & 195.40 & 0.96 & 67.03 \\
\hline 21. & IC 421808 & 94.20 & 3.50 & $27.20 *$ & 4.93 & 3.10 & 63.70 & 1.42 & 61.61 \\
\hline 22. & IC 421315 & $48.00 *$ & 4.20 & $26.90 *$ & 4.86 & 2.94 & 56.45 & $2.30 *$ & 159.27 \\
\hline 23. & IC 421830 & $66.80 *$ & 3.20 & $25.90 *$ & 5.38 & 2.82 & 92.40 & 1.50 & 165.50 \\
\hline 24. & IC 421851 & $63.80 *$ & 3.20 & $24.50 *$ & 5.86 & 3.14 & 119.40 & 1.30 & 160.56 \\
\hline 25. & IC 421856 & 88.00 & $6.00 *$ & $28.80 *$ & 5.84 & 3.22 & 145.30 & 1.48 & 135.32 \\
\hline 26. & IC $38-1$ & 89.40 & $5.80 *$ & 39.40 & 10.76 & 3.32 & 92.80 & 1.48 & 140.24 \\
\hline 27. & $\begin{array}{l}\mathrm{M}-33 \text { Durga } \\
\text { kanchan }\end{array}$ & 57.00 & 4.70 & $24.50 *$ & 8.48 & 3.30 & 63.40 & $2.13 *$ & 126.96 \\
\hline 28. & IC 3773 & 79.60 & $5.80 *$ & 43.60 & 9.78 & 3.30 & 8.50 & $2.70 *$ & 62.07 \\
\hline 29. & IC 9007 / P2 & $71.40 *$ & 4.60 & 39.60 & 9.80 & 3.32 & $58.40^{*}$ & $2.40^{*}$ & 66.57 \\
\hline 30. & IC 10327 & $49.80 *$ & 4.00 & 41.00 & 9.04 & 3.32 & 99.80 & 1.41 & 63.19 \\
\hline 31. & IC 10339 & $45.80^{*}$ & $8.20 *$ & $28.00 *$ & 8.94 & 3.28 & $119.20^{*}$ & $2.32 *$ & 72.62 \\
\hline 32. & IC 10345 & $58.60 *$ & $6.50 *$ & $26.40 *$ & 7.36 & 3.36 & $130.20 *$ & $2.01 *$ & 72.59 \\
\hline 33. & IC 10350 & $66.40 *$ & $10.20 *$ & $29.80 *$ & 8.90 & 3.28 & $101.20^{*}$ & $2.17 *$ & 75.17 \\
\hline 34. & IC 10356 & $58.80 *$ & $6.80 *$ & $30.60 *$ & 9.80 & 3.32 & 98.40 & $2.55^{*}$ & 200.52 \\
\hline 35. & IC 11116 & 69.60 & 4.40 & $28.40 *$ & 9.74 & 3.38 & $123.20^{*}$ & $2.40 *$ & 199.37 \\
\hline 36. & IC 11354 & $60.60 *$ & $5.60 *$ & $31.20 *$ & 9.90 & 3.32 & $104.80 *$ & $2.98 *$ & 190.64 \\
\hline 37. & IC 11357 & $59.00 *$ & $6.00 *$ & $32.40 *$ & 9.50 & 3.34 & 91.40 & $2.41 *$ & 160.25 \\
\hline
\end{tabular}




\begin{tabular}{|c|c|c|c|c|c|c|c|c|c|}
\hline 38. & IC 11388 & $66.40^{*}$ & $7.60 *$ & $31.40^{*}$ & 9.54 & 3.36 & $129.80 *$ & $2.37 *$ & 160.11 \\
\hline 39. & IC 11704 & 73.40 & $8.80 *$ & 37.20 & 9.72 & 3.38 & $155.80 *$ & 1.85 & 155.07 \\
\hline 40. & IC 13496 & 76.00 & $8.40^{*}$ & 41.20 & 9.82 & 3.46 & 99.40 & $2.50^{*}$ & 316.91 \\
\hline 41. & IC 28269 & 93.80 & $6.80 *$ & 36.80 & 10.10 & 3.42 & $146.20^{*}$ & $2.72 *$ & 310.54 \\
\hline 42. & IC 40021 & 72.60 & $6.20 *$ & 40.80 & 9.40 & 3.40 & 88.40 & $2.90 *$ & 307.75 \\
\hline 43. & IC 40040 & $56.00 *$ & $7.20 *$ & 35.80 & 6.16 & 3.38 & 96.00 & $2.17 *$ & 198.10 \\
\hline 44. & IC 51063 & 69.60 & $6.20 *$ & $33.40^{*}$ & 5.50 & 3.34 & 44.30 & 1.76 & 196.32 \\
\hline 45. & IC 421350 & $61.00 *$ & 2.00 & $29.60 *$ & 7.16 & 3.26 & $159.70^{*}$ & 1.50 & 199.44 \\
\hline 46. & IC 421855 & 70.80 & 3.00 & 36.40 & 6.14 & 3.24 & 88.95 & 0.91 & 67.94 \\
\hline 47. & IC 421858 & 73.60 & $6.00 *$ & 34.40 & 8.56 & 3.20 & $104.60^{*}$ & 1.63 & 63.42 \\
\hline 48. & IC 421860 & $53.60 *$ & 3.50 & 36.60 & 6.20 & 3.28 & $115.60^{*}$ & 1.07 & 67.02 \\
\hline 49. & IC 432117 & $65.40 *$ & $5.30 *$ & 43.80 & $12.86^{*}$ & $3.90 *$ & 73.40 & 4.00 & 128.87 \\
\hline 50. & Pusa Naubahar & $51.00 *$ & $8.00 *$ & 41.40 & 11.70 & $4.78 *$ & 72.00 & $2.89 *$ & 140.27 \\
\hline 51. & Thar Bhadavi & 68.60 & 2.00 & 42.80 & 7.78 & $4.78 *$ & 90.40 & 1.61 & 140.45 \\
\hline 52. & GAURI, 1 & $47.40 *$ & 2.50 & $25.00 *$ & 10.60 & 3.60 & 56.90 & 1.50 & 99.19 \\
\hline 53. & $\mathrm{HG}-182$ & 71.80 & $5.00 *$ & 36.00 & 6.10 & 3.18 & 191.80* & 1.32 & 101.40 \\
\hline 54. & $\mathrm{HG}-2-20$ & $51.00 *$ & 2.40 & $33.00 *$ & 6.36 & 3.28 & $143.74^{*}$ & 1.58 & 103.00 \\
\hline 55. & $\mathrm{HG}-75$ & $61.80 *$ & 2.80 & 38.00 & 6.14 & 3.10 & 28.80 & $2.00 *$ & 174.72 \\
\hline 56. & $\mathrm{HG}-875$ & $67.00 *$ & $5.00 *$ & 36.00 & 6.10 & 3.10 & $126.80^{*}$ & 1.80 & 178.45 \\
\hline 57. & $\mathrm{HG}-2-30$ & $61.60 *$ & 3.00 & 39.00 & 8.34 & 3.40 & 92.00 & 1.50 & 177.41 \\
\hline 58. & $\mathrm{HG}-563$ & $57.20 *$ & 1.60 & 34.60 & 6.14 & 3.28 & 10.14 & 1.30 & 176.50 \\
\hline 59. & $\mathrm{HG}-258$ & 90.00 & 2.50 & 38.20 & 5.94 & 3.16 & $150.40^{*}$ & 1.20 & 193.61 \\
\hline 60. & $\mathrm{FS}-277$ & 68.80 & $7.00 *$ & 37.20 & 6.24 & 3.16 & $99.80 *$ & 1.43 & 192.67 \\
\hline 61. & $\mathrm{HG}-870$ & $48.60 *$ & 7.40 & 36.40 & 6.54 & 3.26 & $140.00^{*}$ & 1.90 & 93.53 \\
\hline 62. & $\mathrm{HG}-884$ & 77.60 & 4.60 & $31.50 *$ & 2.42 & 3.16 & 67.90 & 1.98 & 86.88 \\
\hline 63. & HFG -119 & 79.00 & $6.00 *$ & 40.00 & 5.76 & 3.14 & $156.80 *$ & 1.50 & 91.00 \\
\hline 64. & Madurai Local 1 & 71.80 & 2.40 & 35.00 & 11.02 & 3.50 & 90.60 & $3.30 *$ & 130.12 \\
\hline 65. & Madurai Local 2 & $55.40 *$ & 1.80 & 35.00 & $13.30 *$ & 3.26 & 11.02 & $3.48 *$ & 135.31 \\
\hline 66. & Sathur Local 1 & $57.80 *$ & 1.80 & 38.00 & $13.98 *$ & 3.78 & 15.34 & $2.60 *$ & 124.18 \\
\hline 67. & $\begin{array}{l}\text { Thadayampatti } \\
\text { Local } 1\end{array}$ & $60.20 *$ & 2.50 & $32.00 *$ & 11.76 & 3.38 & 95.90 & $2.38 *$ & 144.96 \\
\hline 68. & $\begin{array}{l}\text { Thadayampatti } \\
\text { Local } 2\end{array}$ & 70.60 & 2.00 & $32.00 *$ & $12.75^{*}$ & 3.48 & 84.75 & $3.38 *$ & 133.07 \\
\hline 69. & $\begin{array}{l}\text { Perumalpatti } \\
\text { Local1 }\end{array}$ & 70.40 & 2.50 & $33.00 *$ & 12.22 & 3.62 & 15.42 & $2.94 *$ & 137.87 \\
\hline 70. & $\begin{array}{l}\text { Ramanaickanpatti } \\
\text { Local }\end{array}$ & 88.62 & 3.10 & 37.00 & 10.46 & 3.14 & 35.60 & 1.63 & 156.59 \\
\hline 71. & $\begin{array}{l}\text { Chidamparapuram } \\
\text { Local }\end{array}$ & 73.42 & 3.50 & 40.00 & 6.40 & 3.12 & 25.40 & 1.71 & 149.52 \\
\hline 72. & $\begin{array}{l}\text { Virudhunagar } \\
\text { Local }\end{array}$ & 112.00 & 2.70 & 41.00 & 10.92 & 3.08 & 51.00 & $2.55^{*}$ & 159.44 \\
\hline Mean & & 64.89 & 4.59 & 32.32 & 9.38 & 3.25 & 98.36 & 1.94 & 182.00 \\
\hline SEd & & 1.33 & 0.10 & 0.70 & 2.06 & 0.29 & 0.07 & 0.04 & 3.58 \\
\hline CD & & 2.62 & 0.19 & 1.39 & 3.07 & 0.57 & 0.14 & 0.08 & 7.08 \\
\hline
\end{tabular}


The girth of the fruit was maximum in the genotype Pusa Naubahar and Thar Bhadavi $(4.78 \mathrm{~cm})$ followed by IC $432117(3.90 \mathrm{~cm})$, Thadayampatti local $2(3.78 \mathrm{~cm})$ Perumalpatti local $(3.62 \mathrm{~cm})$ whereas minimum pod girth was recorded in the genotype was IC 370496 (1.98), IC $415106(2.18 \mathrm{~cm})$. The number of fruits per plant has considerable on total yield of fruits pod yield is determined by the fruit weight and number of fruits per plant therefore yield is complex character and it is dependent on its component traits and their inheritance any change in these would reflect on total yield The following accessions namely IC 432117, IC 311407, IC 415125, Thadayampatti local, IC40021 and IC 421855 recorded 73.4, 82.6, $84.4,84.6,88.4$ and 88.9 fruits per plant. IC 11357, HG-2-30, IC 421830, IC 38-1 and Sattur local 1 were recorded IC11357, $\mathrm{Hg}-2-30$, IC421830, IC 38-1 and Sattur local 1 were recorded 90.4, 90.6, 92.0, 92.4 and 92.8 per plant; IC10350, IC421858, IC11354 and IC415108 recorded 101.2, 104.6, 104.8 and 109.2 fruits per plant Among the 72 genotypes IC9007/p2 recorded highest number of fruits per plant (458.4 fruits/plant) and $\mathrm{HG}-258$, IC11704, HFG 119, IC 421350, HG 182 and IC 421805 recorded 150.4, 155.8, 159.7, 191.8 and 195.4 fruits per plant Average individual fruit weight was maximum in IC432117 (4.o g) followed by Madurai local, Thadayampatti local Madurai local 1 and IC373464 (3.48,3.38,3.30 and $3.27 \mathrm{~g} /$ fruit) respectively The yield per plant was maximum in IC 9007/P2 (1100g) IC $28269(1020 \mathrm{~g})$ and IC $373464(643.73 \mathrm{~g})$ followed by IC 432117, IC 402296, IC11354 and IC 11388 (342.53, 312.43, 312.38 and $308.27 \mathrm{~g} /$ plant) Whereas IC 329062, IC370509, IC 415106 recorded minimum fruit yield of 63.67, 64.15 and 66.53 g/plant (Table 1).
Lohesha and Shiv Shankar (1990) reported that pod weight and total yield was strongly associated with plant dry weight, number of leaves and number of cluster Singh et al., (1990) observed that complementary gene action for number of branches and duplicate gene action for pod clustering pattern Estimated per se performance, heritability and genetic gain were high for pods per plants and pod weight (Taneja et al., 1995) and these characters were used for selection in breeding program.

\section{References}

Gibson, A. and Balakrishnan, R. 1990. Variability studies in Cluster bean. (Cyamopsis tetragonoloba (L.) Taub.). South Indian Horticulture, 38(6): 311 314.

Lokesha, R and Shivshankar, G. 1990. Analysis of genetic Variability and Character Association in Cluster Bean. Mysore Journal of Agricultural Sciences, 24(3): $316-320$.

Rakesh Pathak, S. K., Singh, V., Manjit Singh and Henry, A. 2010. Molecular assessment of genetic diversity in Cluster bean (Cyamopsis tetragonoloba (L.) Taub.) genotypes. Journal of Genetics, 89(2): 243 - 246.

Singh, J.V., Lodhi, G.P., Saini, M.L. and Singh, V.P. 1990. Inheritance of branching and pods clustering pattern in Cluster bean (Cyamopsis tetragonoloba (L.) Taub.). Crop Research, 3(1): 93 - 95.

Taneja, K.D., Bishoi, O.P., Rao, V.U.M. and Niwas, R. 1995. Effect of environment on growth and yield of Cluster bean. Crop Research, 9(1): 159-162.

\section{How to cite this article:}

Premalakshmi, V., T. Arumugam, N. Deepa Devi and Ramesh Kumar, S. 2017. Development of New Variety in Cluster Bean (Cyamopsis tetragonoloba). Int.J.Curr.Microbiol.App.Sci. 6(4): 25412545. doi: https://doi.org/10.20546/ijcmas.2017.604.296 